\section{P-72 MOVING UP THE 'TRIANGLE' TO CREATE A RESEARCH ACTIVE HOSPICE}

Sarah M McGhee, Giovanna I Cruz, Dianne Corrin, Cheryl Young, Lottie Morris, Hollie Quaye. Hospice Isle of Man, Strang, Isle of Man

\subsection{6/bmjspcare-2018-hospiceabs.97}

Background There is a welcome and appropriate movement, encouraged by Hospice UK, towards increasing the role of research in palliative and end of life care.

Aim To enable Hospice Isle of Man to reposition as a Research Active Hospice.

Methods A survey was undertaken to ascertain the current level of research interest and awareness in Hospice Isle of Man.

Results to date The survey was completed by 25 staff and 45 volunteers. The majority $(88 \%)$ of clinical staff had some research training but most had no recent opportunity to engage in research; over $70 \%$ agreed that research added value to their daily work but only $40 \%$ were confident in their critical appraisal skills. As a result further training e.g. in critical appraisal skills within journal clubs is being initiated. Amongst the volunteers 22\% were identified to have research skills but very few were actively engaged in research. These volunteers will be invited to participate in future projects. Funding from the Scholl Foundation and the Manx Lottery Trust has enabled research staff to be recruited and has facilitated protected time for some clinical staff to participate in research with peer support and skill sharing in, for example, developing outcome measurements, appropriate analyses and interpretation of data and considering how the value of routine data can be improved. Initial uncertainty about the role of research and researchers is gradually being replaced with appreciation of their support in enhancing research awareness and activity among staff.

Conclusion Hospice Isle of Man had a low level of research activity however a step-change has been achieved by utilising existing skills and interests of the clinical staff. Key drivers were obtaining funding for protected research time for key clinical staff and employing experienced researchers to support and complement the clinical staff to develop an active research culture.

\section{P-73 ARE WE ACHIEVING PREFERRED PLACE OF DEATH (PPD) IN PATIENTS REFERRED TO OUR PALLIATIVE CARE SERVICE?}

Victoria Otway. Countess Mountbatten Hospice, Southampton, UK

\subsection{6/bmjspcare-2018-hospiceabs.98}

Aim To review whether a cohort of patients referred to the Countess Mountbatten Palliative Care service achieved their preferred place of death (PPD).

Method A clinical notes review of the first 100 patients referred to the service from 1st June 2017 specifically looking at preferred place of death, actual place of death and, if relevant, reasons PPD wasn't achieved. Length of time known to service and likelihood of achieving PPD were also studied.

Results Of the 100 patients studied 35 had PPD of Home, 25 had PPD of Hospice, three had PPD of acute hospital, 27 PPD remained unknown and 10 had no preference.
PPD of Home was achieved in 17 of the 35 patients (49\%). PPD of Hospice was achieved in 22 of the 25 patients $(88 \%)$ and PPD of acute Hospital was achieved in all three patients (100\%). Patients known to the service between 2 to 12 weeks were the most likely to achieve their PPD (64\%).

Discussion Lack of care and equipment at home, carer distress and difficult symptom control were the main reasons for not achieving PPD of Home. Reasons for not achieving PPD of Hospice included rapid deterioration making patient transfer inappropriate. Where PPD was unknown, most patients were only known to the service for a short period before death and the majority of patients were acutely unwell and PPD discussion was felt to be inappropriate.

Conclusions Rapid access to care and equipment in the home could result in more patients achieving their PPD. Increased community support and a hospice at home service could potentially provide this.

\section{P-74 HOSPICE CONTRIBUTION TO THE UK NIHR NATIONAL CLINICAL RESEARCH PORTFOLIO: 2018 UPDATE}

Sam H Ahmedzai, Shamaila Anwar, Jonathan Gower, Matt Seymour. NIHR Clinical Research Network: Cancer Cluster, Leeds, UK

\subsection{6/bmjspcare-2018-hospiceabs.99}

The National Institute for Health Research (NIHR) is responsible for funding, delivery and monitoring of clinical research studies in the NHS. The main subset of NHS studies relevant to hospices is categorised under 'Supportive and Palliative Care' and 'Psychosocial Oncology and Survivorship' (SPC/POS). In the NIHR, the Clinical Research Network (CRN): Cancer Cluster is responsible for overseeing the delivery of this subset within the NIHR portfolio, not only in cancer but also extending to advanced non-malignant disease.

Since January 2018, non-NHS sites such as hospices are also officially recognised as sites for NHS-based studies. Of course, hospices have been involved and indeed leading in clinical research for many years - the Cancer Cluster office is now regularly tracking this important contribution. In 20162017, there were a total of 49 studies open in the SPC/POS group, of which 43 were actively recruiting. There is a balanced mixture of observational and interventional research. Participating sites included 22 hospices and 125 hospital trusts. (Some hospices may be listed under their local trust name.) There is wide variation across England and the devolved nations with respect to participation in these studies.

Funding for studies relevant to hospices came mainly from NIHR and other major charities, including Marie Curie, Macmillan, research councils. A few studies have commercial sponsorship.

The NIHR CRN is committed to helping the hospice sector to increase its access to clinical trials, through the NIHR Charities Consortium. It will do this by disseminating information and supporting the research workforce, working with Hospice UK. The Consortium held its first national conference on hospice and community-based research in April 2018. This will become an annual event and there will be prizes to reward hospices and other SPC/POS research teams for making significant contributions to the national NIHR portfolio. 